\title{
Error Analysis for Networks Supplying Unbalanced Nonlinear Load
}

\author{
${ }^{1}$ S. K.Bhuyan, ${ }^{2}$ A.K.Baliarsingh, ${ }^{3}$ D.P.Dash, ${ }^{4}$ A.K Sahu, ${ }^{5}$ A.K Mangaraj \\ ${ }^{1,}$ Dept. of Electrical Engg. BIET, Bhadrak ${ }^{2,3 \& 5}$ Dept. of Electrical Engg. OEC.BBSR, ${ }^{4}$ Dept. of Electrical Engg. \\ OSME,Kounjar
}

\begin{abstract}
Power quality is the set of parameters defining the properties of power supply delivered to the users in normal operating conditions in terms of continuity of supply and characteristics of voltage (magnitude, frequency, symmetry, waveform etc.). The increased requirements on supervision, control, and performance in modern power systems make power quality monitoring a common practice for utilities. Power signal waveforms are not clean $50 \mathrm{~Hz}$ sine waves. Waveform distortion is an important aspect of power quality. In a power system, harmonics, transient impulses and noise are mainly responsible for waveform distortion. Harmonic analysis is an important part of power quality monitoring system
\end{abstract}

\section{INTRODUCTION}

The unbalanced loading of the three-phase supply has detrimental effects, such as the underutilization of the power supply equipment and the overloading of neutral conductors with the fundamental frequency in addition to harmonic currents. Finally, as is well known, a phase displacement between the corresponding voltages and currents indicates both a low utilization of the generation and distribution equipment and increased line losses for the same power consumption level. The ever-growing proliferation of power switching devices for source conditioning and motion control in single-phase and three-phase modern industrial applications has increased the eventuality of unbalanced currents, unacceptable harmonic levels, and poor power factor in threephase distribution systems. It is remarked that a low power transfer quality could be caused either by the power supply (through unbalanced supply voltages and/or voltage harmonics), by the load (nonlinear and/or unbalanced load), or by both [10]. The newly suggested PQF has the advantage of evaluating the power quality at any selected point in a distribution network by means of a single indicator rather than separately comparing a multitude of factors with their recommended values, which thus considerably simplifies the rate structures. The measuring device offers the additional feature of distinctly measuring the different aspects affecting the power quality, thus identifying any specific power quality aspect that needs attention.

\subsection{QUANTITATIVE FORMULATIONS OF POWER QUALITY ASPECTS}

\subsubsection{Total Current and Voltage Harmonic Distortion}

The total harmonic distortions of the voltage and current (VTHD and ITHD, respectively) for a single phase (or poly-phase balanced networks) have been conventionally defined in the literature [1], [7] as

$$
\begin{aligned}
& {[\text { VTHD }]^{2}=\frac{\sum_{h \neq 1}^{n} v_{h}^{2}}{V_{1}^{2}}} \\
& {[I T H D]^{2}=\frac{\sum_{h \neq 1}^{n} I_{h}^{2}}{I_{1}^{2}}}
\end{aligned}
$$

Where $V$ and $I$ denote the rms values, and 1 and $h$ denote the fundamental and the harmonic order, respectively. To account for the fact that higher order harmonic currents cause greater losses than lower order harmonics of the same amplitude, the harmonic-adjusted total voltage and current harmonic distortions are, respectively, defined as

$$
\begin{aligned}
& {[\text { VTHD }]_{H-A}^{2}=\frac{\sum_{k \neq 1}^{n}\left[C_{h} V_{h}\right]^{z}}{V_{1}^{z}}} \\
& {[I T H D]_{H-A}^{2}=\frac{\sum_{k \neq 1}^{n}\left[D_{h} I_{h}\right]^{z}}{I_{1}^{z}}}
\end{aligned}
$$

Where $C_{h}$ and $D_{h}$ are the appropriate weighting factors (greater than 1) that monotonically increase with the harmonic order $h$. Various mathematical expressions, which are by no means exhaustive, for these weighting factors, as a function of the harmonic order $h$, are suggested in [6]. An extension of the definitions (1) and (2) to unbalanced poly-phase networks has been suggested in [7]. To this end, a single "equivalent" harmonic rms 
voltage $V_{e H}$ and a single "equivalent" harmonic rms current $I_{e H}$ for the three-phase system $a, b$, and $c$ are defined as

$$
\begin{aligned}
& V_{e H}^{2}=\sum_{h \neq 1} \frac{V_{a h}^{2}+V_{b h}^{2}+V_{c h}^{2}}{3} \\
& I_{e H}^{2}=\sum_{h \neq 1} \frac{I_{a h}^{2}+I_{b h}^{2}+I_{c h}^{2}}{3}
\end{aligned}
$$

It is proposed to generalize definitions (5) and (6) and separately weigh individual harmonics similar to (3) and (4), which yield the adjusted harmonic equivalent values

$$
\begin{aligned}
& V_{e H-A}^{2}=\sum_{h \neq 1} C_{h}^{2} \frac{V_{a h}^{2}+V_{b h}^{2}+V_{c h}^{2}}{3} \\
& I_{e H-A}^{2}=\sum_{h \neq 1} D_{h}^{2} \frac{I_{a h}^{2}+I_{b h}^{2}+I_{c h}^{2}}{3}
\end{aligned}
$$

The first introduced quality aspects $\mathrm{QA}_{1}$ and $\mathrm{QA}_{2}$ are identified with the total harmonic distortions VTHD and ITHD for a three-phase unbalanced system and are defined as

$$
\begin{aligned}
& Q A_{1}=V T H D=\frac{V_{\alpha H-A}}{V_{\alpha 1}} \\
& Q A_{2}=I T H D=\frac{I_{\alpha H-A}}{I_{\alpha 1}}
\end{aligned}
$$

Definitions (5)-(8), (11), and (12) satisfy the particular requirement that the total losses in the equivalent balanced three phase system be equal to those in the actual system considered. The underlying assumptions are the following: 1) The line and equipment losses for a certain frequency are proportional to either the square of the voltage or the square of the current; and 2) the line and equipment parameters are symmetrical.

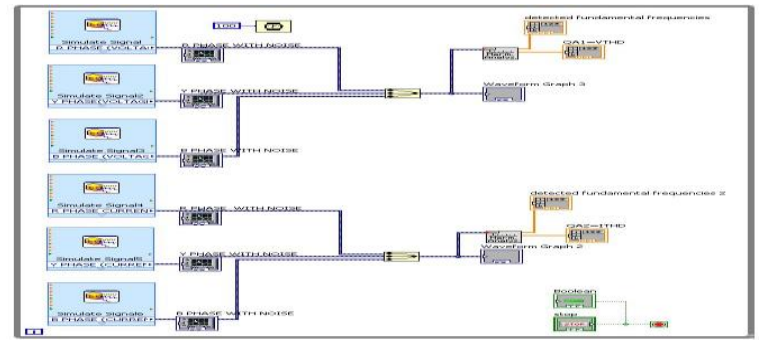

Fig. 1.1 Simulation model

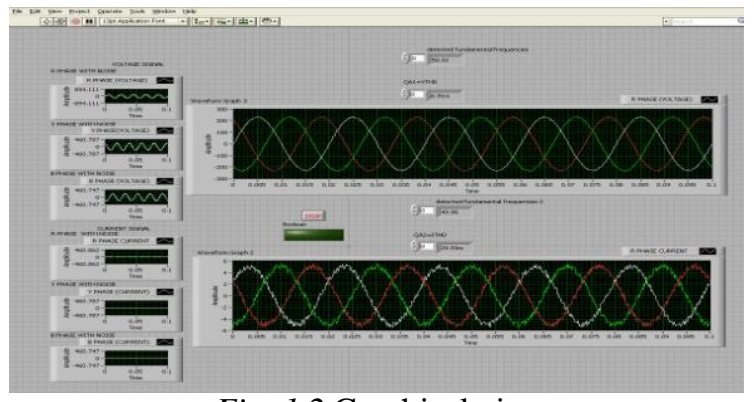

Fig. 1.2 Graphical view

\subsubsection{Degree of Unbalance}

As is well known, the unbalanced voltages and currents in polyphase networks affect the quality of power transfer in many aspects, such as increased line losses for the same power transfer level, extra rotating losses in drives, and overloading of neutral conductors in four-wire distribution systems.

Applying the theory of symmetrical components [8], an unbalanced three-phase sinusoidal voltage system [Va, $V b, V c]$ can be decomposed into a positive-sequence three-phase balanced system $V+$, a negative-sequence system $V-$, and a zero sequence system $V 0$ according to

$$
\begin{aligned}
& \underline{\mathrm{V}^{+}}=\frac{1}{\sqrt{a}}\left(\underline{V_{\alpha}}+a \underline{V_{b}}+a^{2} \underline{V}_{c}\right) \\
& \underline{\mathrm{V}}==\frac{1}{\sqrt{a}}\left(\underline{V_{\alpha}}+a^{2} \underline{V}_{c}+a \underline{V_{c}}\right)
\end{aligned}
$$


$\underline{\mathrm{V}^{\mathrm{o}}}=\frac{1}{\sqrt{a}}\left(\underline{V_{\alpha}}+\underline{V_{b}}+\underline{V_{c}}\right)$

Where $V$ denotes the phasor of $V$, and the factor $a=\exp (j 2 \pi / 3)$. From (11)-(13), the equivalent voltage can be expressed as [12]

$V_{\text {E1 }}^{2}=\underline{\mathrm{V}}^{+2}+\underline{\mathrm{V}} \mathbf{-}^{2}+\underline{\mathrm{V}}^{\mathrm{0} 2}$

Identical considerations apply to the three-phase currents where $V$ is exchanged by $I$. For the purpose of assessing the degree of unbalance, the fundamental frequency phasors of the phase voltages are substituted in (13) to determine $V+, V-$, and $V 0$. The symmetrical components of the currents $I+, I-$, and $I 0$ at the fundamental frequency are similarly derived. The next introduced quality aspects $Q A 3$ and $Q A 4$ are identified with the newly proposed voltage and current unbalance factors VUNB and IUNB defined as

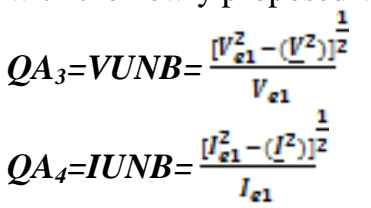

The voltages and currents in definitions (15) and (16) denote absolute values of the corresponding phasors as defined in (11) and (12) and (7) and (8). For balanced three phase voltages and currents, it can readily be shown from (13) and (14) that $V^{-}, V_{0}, \mathrm{I}-$, and $I_{0}$ are equal to $0, V e 1=V+, I e 1=I+$, and hence, $Q A 3=Q A 4$ $=0$.

\subsubsection{Phase Displacements between Corresponding Fundamental Voltage and Currents}

In a power transfer context, a phase displacement between the fundamental frequency voltage and current in a source or load indicates a less than full utilization of the generation and distribution equipment, as well as increased line losses for the same level of power transfer. This has been traditionally expressed through the concept of a power factor $K$ that is defined in single-phase situations as the ratio between the average active power $P$ and the apparent power $S$, with the latter being the product of the rms values of the voltage and current. However, in a non sinusoidal situation, the concept of power factor above has been proved to be misleading [9], [10]. Moreover, a universally accepted definition of the power factor in polyphase situations has been lacking and hotly debated for almost a century [11], [12].

This stems from the different definitions and interpretations of the apparent power [13], [14] in these situations. It should be noted in this context that only the instantaneous power, i.e., the product of the instantaneous values of the voltage and current, has the physical meaning of power.

The conventional active power $P$ derives its physical relevance from being the time average of the instantaneous power. In contrast, the apparent power $S$ has no physical meaning since it does not consider the corresponding values of the voltage and current at each instant. Only in the special case when the instantaneous voltage bears a constant ratio to the instantaneous current does the apparent power equal $P$ and, hence, acquires the physical meaning of the latter [10].

All the inadequacies and ambiguities above can be circumvented when the physical entity directly responsible for a poor utilization of the power capacity at the mains fundamental frequency is considered. This entity is the phase difference between the fundamental frequency voltage and current at the terminals of the source or load. To reflect this aspect of power quality, a new factor, which is designated as the orthogonal current factor (OCF), is suggested as follows:

$$
\begin{array}{ll}
\boldsymbol{O F C}=\frac{I 1 a \operatorname{Sin} \varphi 1 a+I 1 b \operatorname{Sin} \varphi 1 b+I 1 c \operatorname{Sin} \varphi 1 c}{I 1 a+I 1 b+I 1 c} & \ldots .(\text { Eq 17) } \\
\boldsymbol{Q A} \boldsymbol{A}_{5}=|\boldsymbol{O C F}| & \ldots .(\text { Eq 18) }
\end{array}
$$

Where $\mathrm{I}_{1} \mathrm{a}, \mathrm{I}_{1} \mathrm{~b}$, and $\mathrm{I}_{1} \mathrm{c}$ denote the rms values of the phase currents at the fundamental frequency, and $\varphi_{1} \mathrm{a}, \varphi_{1} \mathrm{~b}$, and $\varphi_{1} \mathrm{c}$ denote the phase differences between the fundamental frequency components of the corresponding phase voltages and currents. The rationale behind definition (17) is that the orthogonal current component I $\sin \varphi$ of a phase current with respect to the corresponding phase voltage is directly related to the effort required to ideally reduce the phase $\varphi$ to 0 . For example, in a single-phase situation, the capacitance required to connect in parallel to an inductive load to bring $\varphi$ to 0 is given by

$$
C=I \sin \varphi / \omega v
$$


Where $\omega$ is the fundamental angular frequency. In particular, instances of lagging and leading orthogonal components in different phases may partially or totally cancel each other in (17). This means however that the poly-phase system is strongly unbalanced, and the unbalance quality aspects in (15) and (16) would prominently reflect it.

\section{PQF}

A new single measurable indicator (i.e., PQF) is proposed to integrally reflect the different power quality aspects formulated in the last section. This is expressed as

Where $\mathrm{w}_{\mathrm{i}}$ 's are the weighting factors that sum up to 1 , and $\mathrm{QA}_{\mathrm{i}}$ 's are the different quality aspects formulated in Section II. A balanced loaded network with sinusoidal currents and voltages, and zero phase displacements, yields an ideal PQF of unity. Conversely, a low value of PQF would indicate a low degree of utilization of the power capacity of the source and/or a high level of harmonics and/or a high degree of unbalance between the phases with the contribution of each aspect well defined and measurable, as illustrated later. Ideally, the particular values of the weighting factors wi and the recommended value of PQF would be unanimously agreed upon by the power generating and distribution community so that the relative importance of the different quality aspects enumerated above is judiciously reflected. This does not exclude of course the possibility that the set of weighting factors be different for different types of consumers. Particular recommendations for the weighting factors and the minimum tolerated PQF would naturally be supported by economic studies. Examples of basic considerations in such studies are, on one hand, the cost of harmonic filters, reactive power compensators, balancing circuits, and the like, and on the other hand the economic gains resulting from a highquality power transfer, such as a high degree of utilization of the source capacity, prolonged life of system components, and minimization of system outages due to resonance phenomena caused by harmonics.

These considerations are similar to those required to specify acceptable limits to the voltage and current distortions [16] or the minimum tolerated power factor in its classic sinusoidal sense, deviations from which have been commonly penalized in rate structures. The notable features of definition (20) are the following. 1) It gives a quick assessment of the power transfer quality at the selected point of the supply. 2) The definition does not make use of controversial or misleading quantities such as apparent power and power factor in non-sinusoidal situations. 3) It can be expanded to include additional power quality aspects (QA's), such as flicker that are appropriately defined in terms of their frequency and/or amplitudes. 4) The weighting factors can be flexibly selected to reflect technical and economic priorities in different environments.

Detail Procedure for determining Quality aspects in Lab view Environment:

- 3 phase voltage and current(R, Y, \& B) with noise are fed to the Merge (Merges two or more signals into a single output).

- We can see the wave shape of individual R, Y and B phases which are 120 degree electrical apart from each other are connected to the waveform graph displays. The waveform graph plots only single-valued functions, as in $y=\mathrm{f}(x)$, with points evenly distributed along the $\mathrm{x}$-axis, such as acquired time-varying waveforms. Wave form graph have its own front panel.

- The output of merged signal can be displayed by single waveform graph indicator, where we can see the combined 3 phase wave of current and voltage.

- Then the output of merged signal fed to the Harmonic Analyzer

Harmonic Distortion Analyzer 1 Chan

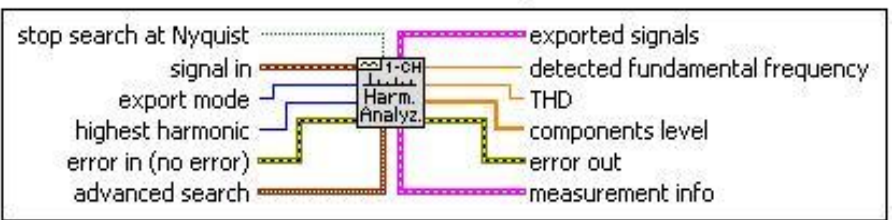

Harmonic Distortion Analvzer N Chan

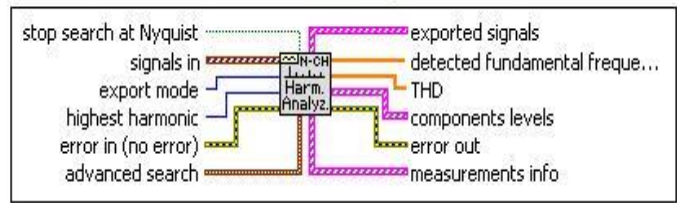


Fig. 1.3 shows the basic harmonic analyzer which is a very powerful tool in Lab View Takes a signal in and performs a full harmonic analysis, including measuring the fundamental frequency tone and harmonics, and returning the fundamental frequency, all harmonic amplitude levels, and the total harmonic distortion (THD).

From harmonic analyzer we can take THD and Detected Fundamental frequency. The output of harmonic analyzer is fed to the indicator which shows the THD and detected fundamental Frequency. Detected fundamental frequencies are an array containing the detected fundamental frequency resulting from searching the frequency domain for each waveform. THD returns an array containing the measured total harmonic distortion for each waveform.

A while loop is used to continue the process which repeats the sub diagram inside it until the conditional terminal, an input terminal, receives a particular Boolean value. The Boolean value depends on the continuation behavior of the While Loop. Right-click the conditional terminal and select Stop if True or Continue if True from the shortcut menu.. The While Loop always executes at least once. If we select a While Loop on the Execution Control Express Vis and Structures palette and place it on the block diagram, a stop button also appears on the block diagram and is wired to the conditional terminal.

As from Voltage merged signal we get $V_{\text {thd }}$, which is our Quality Aspect 1 . Similarly from $3 \square$ current merged Signal we get $\mathrm{I}_{\text {thd }}$, which is our Quality Aspect 2.Similarly other quality aspects can be determined accordingly.

\subsection{MEASUREMENT OF THE PQF-THE POWER QUALITY METERUSING LAB VIEW}

Third harmonic voltage with magnitude of 0.05 with white noise having magnitude 0.02 is added with the normal voltage wave having peak magnitude 1 of phase A, phase B \& phase C.

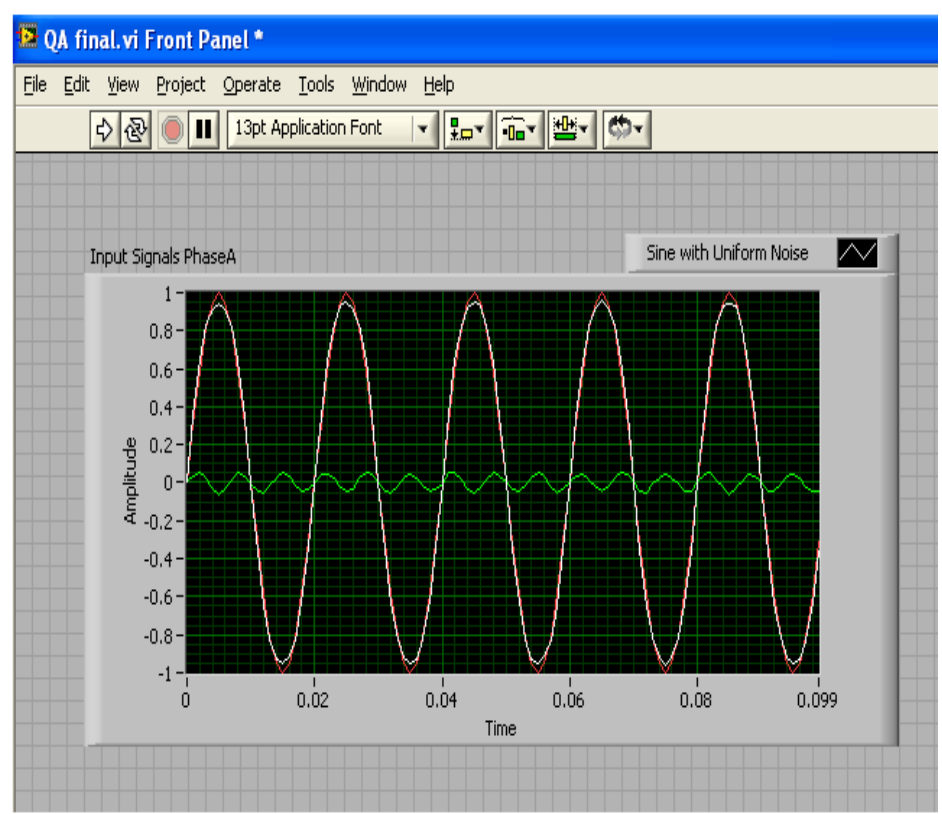

Fig. 2.2 Front panel presenting simulated Phase A input voltage

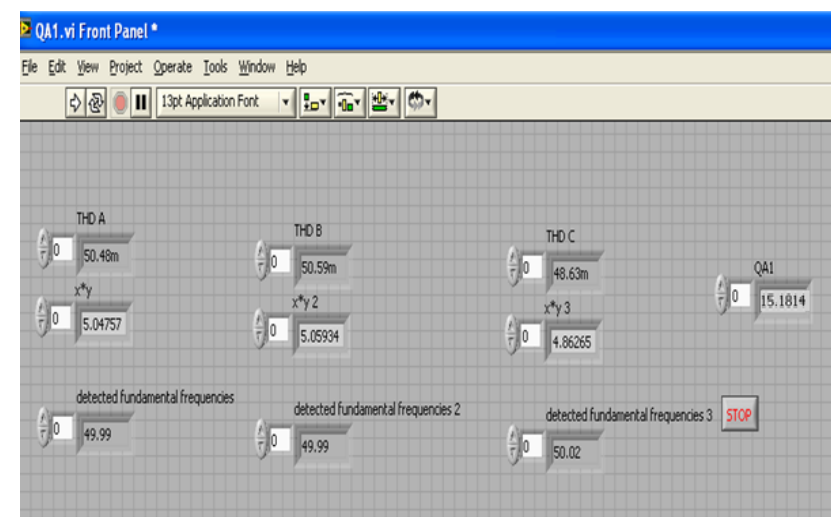

Fig. 2.3 Front panel presenting simulated output of $Q A_{1}$ 
From above Fig. 2.3 the QA1= 15.1814.

Third harmonic current with magnitude of 0.08 with white noise having magnitude 0.02 is added with the normal current wave having peak magnitude 1 of phase A, phase B \& phase C.

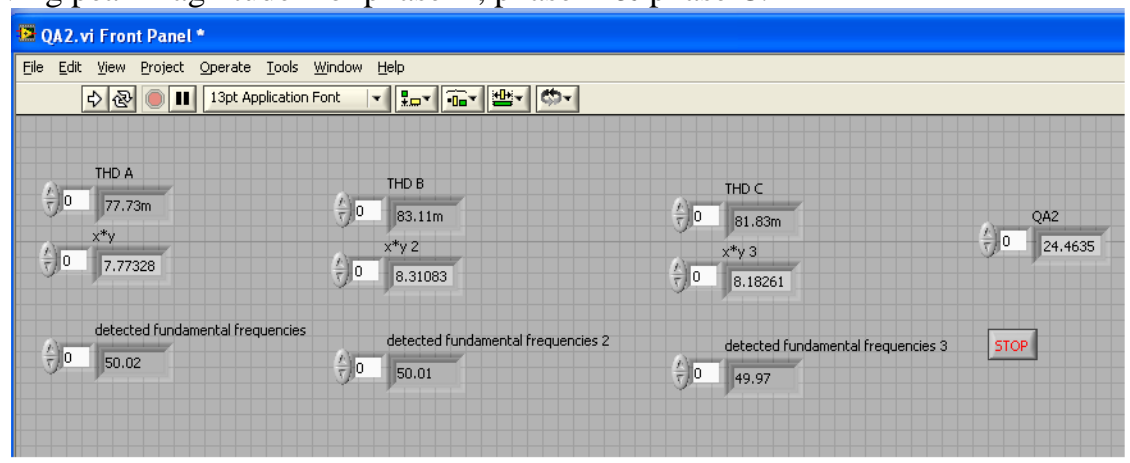

Fig. 2.4 Front panel presenting simulated output of $Q A_{2}$

From above Fig. 2.4 the QA2= 24.4635.

Third harmonic voltage with magnitude of 0.08 with white noise having magnitude 0.02 is added with the normal voltage wave having peak magnitude 1 of phase $\mathrm{A}$.

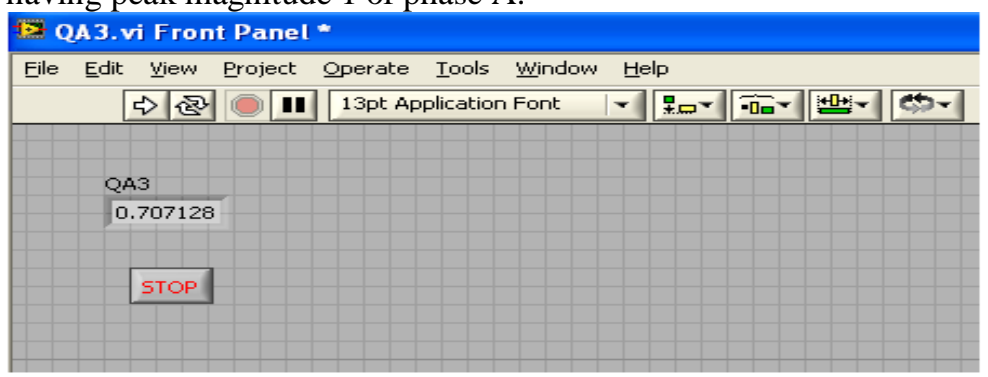

Fig. 2.5 Front panel presenting simulated output of $Q A_{3}$

From the above Fig. 2.5 Front Panels Diagram QA3=0.707128.

Magnitude of 0.08 with white noise having magnitude 0.02 is added with the normal current wave having peak magnitude 1 of phase $\mathrm{A}$.

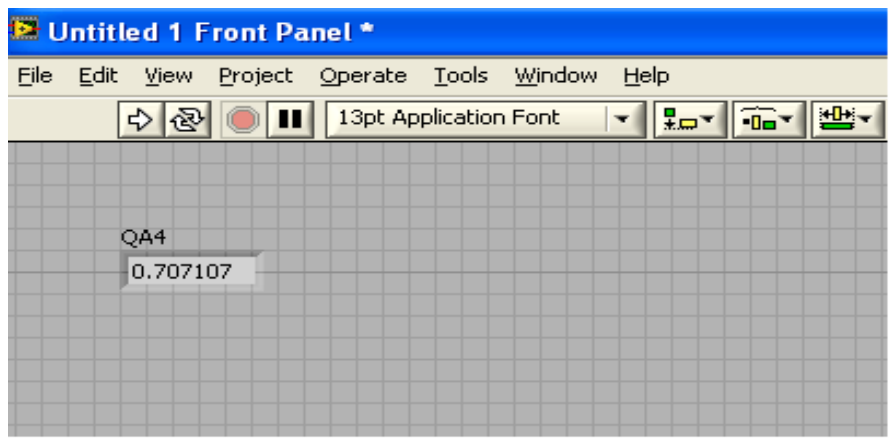

Fig. 2.6 Front panel presenting simulated output of $Q A_{4}$

From the above Fig. 2.6 Front Panels Diagram $\mathrm{QA}_{4}=0.707107$. 


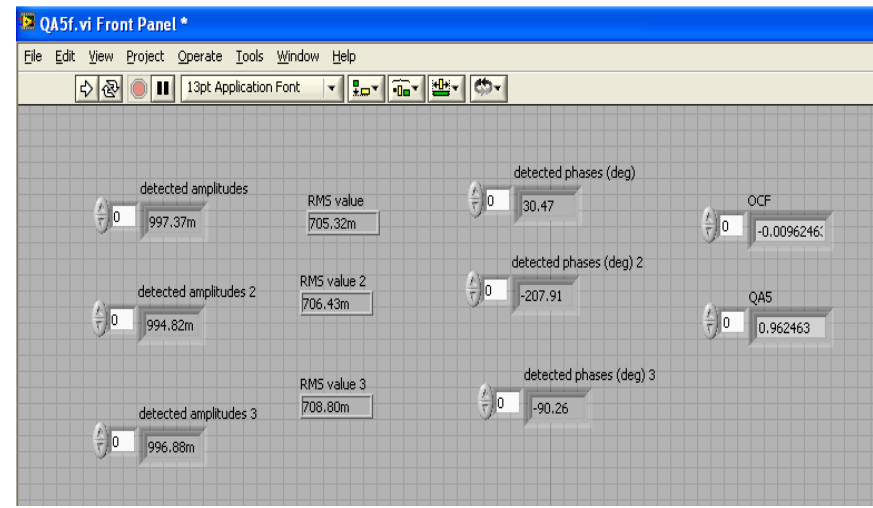

Fig. 2.7 Front panel presenting simulated output of $Q A_{5}$

The above Front Panel Shows RMS values of all the phases of Voltage, the QA5= OCF=0.962463.

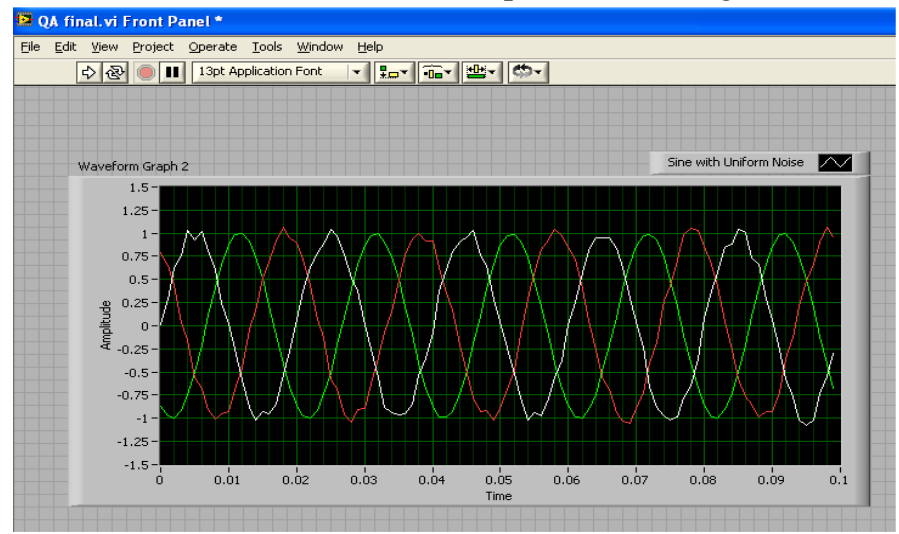

Fig. 2.8 Front panel presenting three phase waves with harmonic destruction having phase displacement of 30,150, -90 degree.

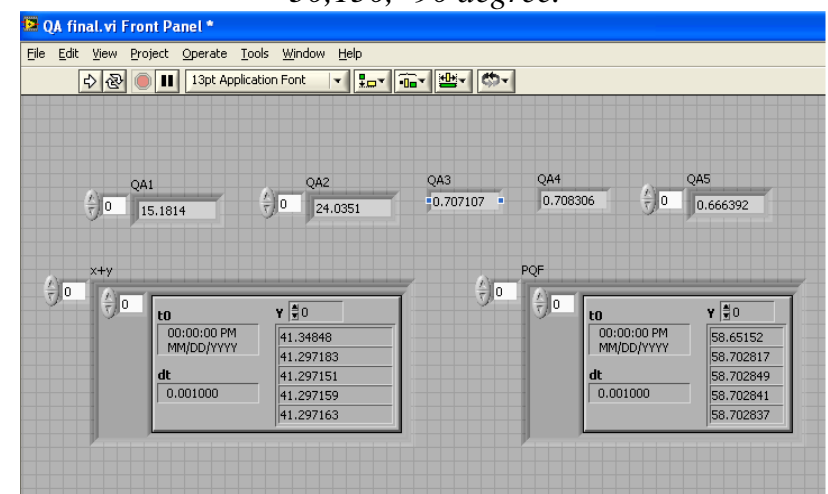

Fig. 2.9 Front panel presenting simulated output $P Q F$

From the above Fig 2.9 Front Panel Diagram the QA1=15.1814, QA2=24.0351, QA3=0.707107, QA4=0.708306 and QA5 $=0.666392$. From the above Front panel Diagram the total Power quality Factor $(\mathrm{PQF})=58 \%$ approx. Third harmonic voltage with magnitude of 0.001 with white noise having magnitude 0.02 is added with the normal voltage wave having peak magnitude 1 of phase A, phase B \& phase $\mathrm{C}$. 


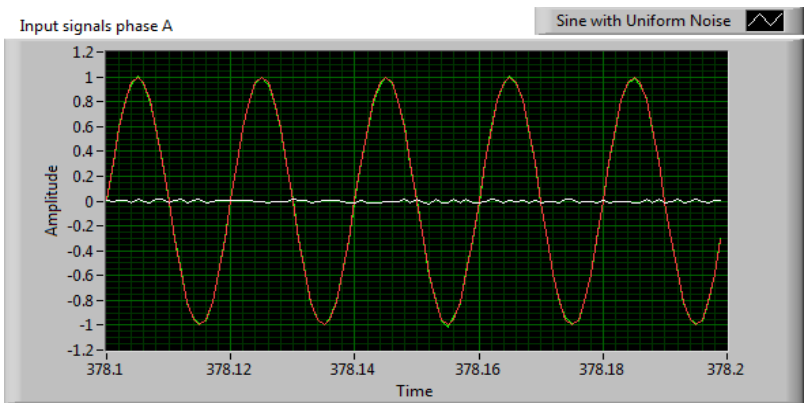

Fig. 2.10 Front panel presenting simulated Phase A input voltage with very low harmonics

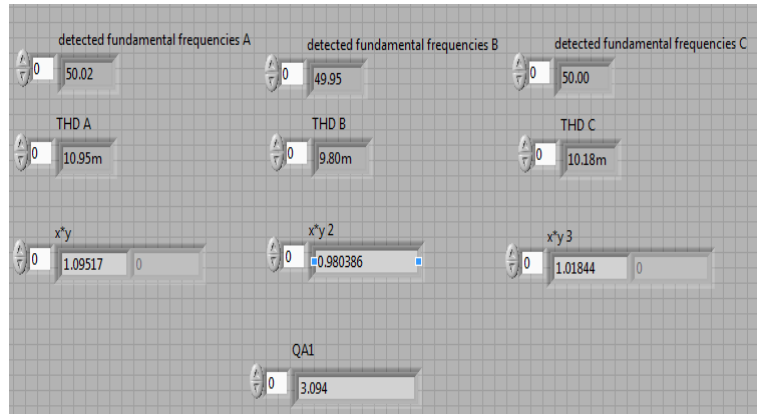

Fig. 2.11 Front panel presenting simulated output QA1 with very low harmonics

Third harmonic current with magnitude of 0.0001 with white noise having magnitude 0.02 is added with the normal voltage wave having peak magnitude 1 of phase A, phase B \& phase C.

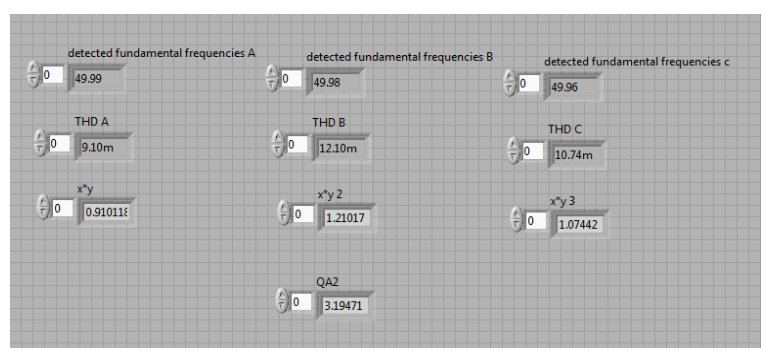

Fig. 2.12 Front panel presenting simulated QA2 with very low harmonics

Third harmonic voltage with magnitude of 0.08 with white noise having magnitude 0.02 is added with the normal voltage wave having peak magnitude 1 of phase A, phase B \& phase $\mathrm{C}$.

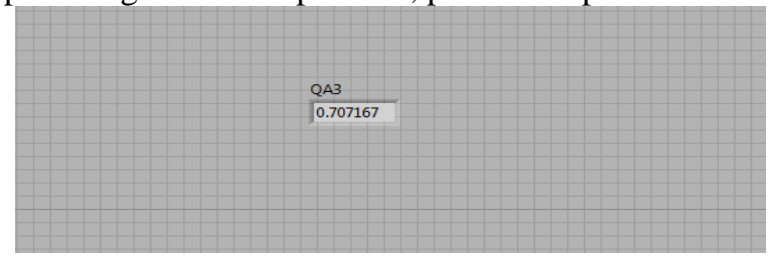

Fig. 2.13 Front panel presenting simulated QA3 with very low harmonics

Third harmonic current with magnitude of 0.08 with white noise having magnitude 0.02 is added with the normal current wave having peak magnitude 1 of phase A, phase B \& phase C.

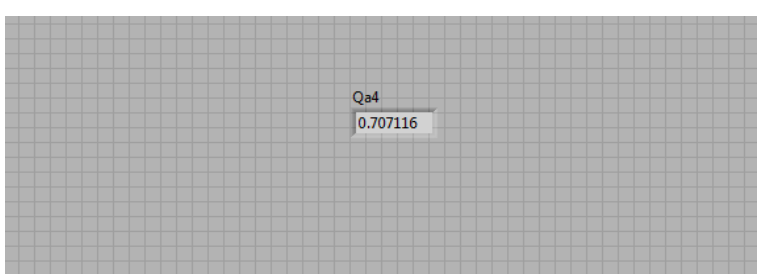

Fig. 2.14 Front panel presenting simulated QA4 with very low harmonics 


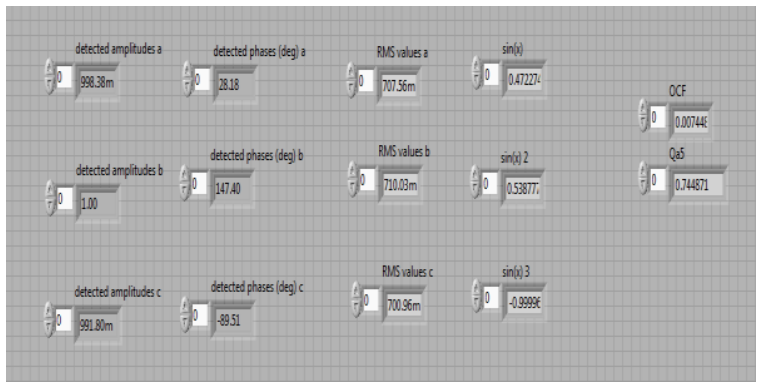

Fig. 2.15 Front panel presenting simulated QA5 with very low harmonics

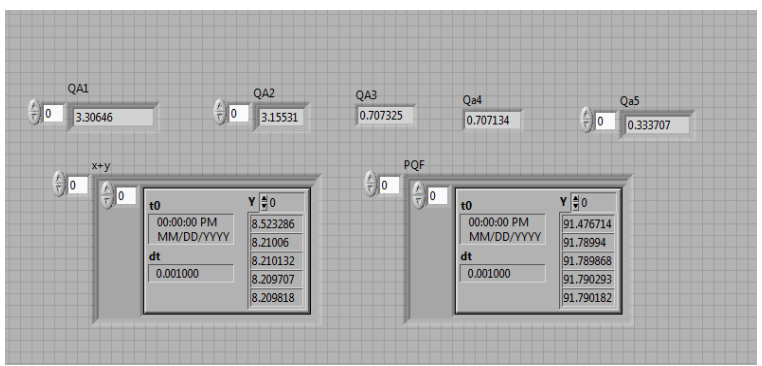

Fig. 2.16 Front panel presenting simulated $P Q F$ with very low harmonics

From the above Fig. 2.16 Front Panel Diagram the QA1=3.30646, QA2=3.15531, QA3=0.707325, QA4=0.707134 and QA5=0.333707. From the above Front panel Diagram the total Power quality Factor $(\mathrm{PQF})$ $=91 \%$ approx.

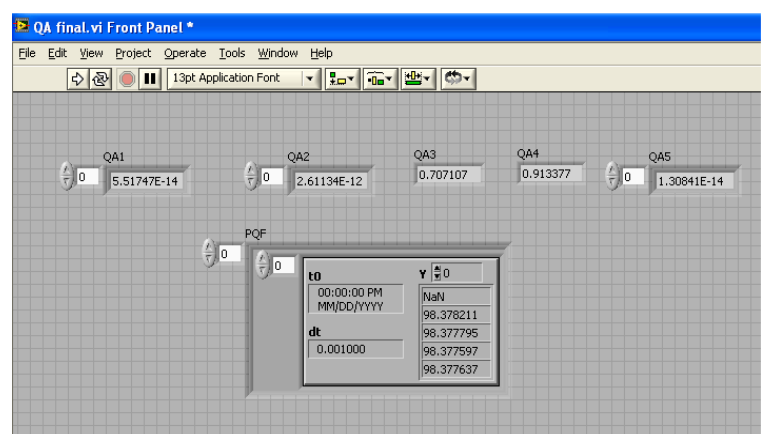

Fig 2.17 Front panel presenting simulated output PQF for healthier phases

Note- Basically PQF depends upon current harmonic rather than voltage harmonic, \& depends upon the nonlinear load. If the harmonic is reduced then Power Quality Factor is increased \& vice versa.

\section{REFERENCES}

[1] V. E. Wagner, J. C. Balda, T. M. Barnes, A. E. Emannuel, R. J. Ferraro,D. C. Griffith, D.P. Hartmann,W. F. Horton,W. T. Jewell, A. McEachern,D. J. Phileggi, and W. E. Reid, "Effectsof harmonics on equipment," IEEE Trans. Power Del., vol. 8, no. 2, pp. 672-680, Apr. 1993.

[2] R. D. Henderson and P. J. Rose, "Harmonics: The effects on power quality and transformers," IEEE Trans. Ind. Appl., vol. 30, no. 3, pp. 528-532, May/Jun. 1994.

[3] IEEE Recommended Practices and Requirements for Harmonic Control in Electrical PowerSystems, IEEE Std. 519-1992, 1993.

[4] A. McEachern, W. M. Grady, W. A. Moncrief, G. T. Heydt, and M. McGranaghan,"Revenue and harmonics: An evaluation of some proposed rate structures," IEEE Trans.Power Del., vol. 10, no. 1, pp. 474-482, Jan. 1995.

[5] "Practical definitions for powers in systems with nonsinusoidal waveforms and unbalancedloads: A discussion," IEEE Trans. Power Del., vol. 11, no. 1, pp. 79-101, Jan. 1996.

[6] C. L. Fortescue, "Method of symmetrical coordinates applied to the solution of polyphase networks," Trans. AIEE, vol. 37, pt. II, pp. 1027-1140, 1918.

[7] D. Sharon, "Reactive power definitions and power factor improvement in nonlinear systems,"Proc. Inst. Electr. Eng., vol. 120, no. 6, pp. 704-706, Jun. 1973. 
[8] D. Sharon, "Power factor definitions and power transfer quality in nonsinusoidal situations,"IEEE Trans. Instrum. Meas., vol. 45, no. 3, pp. 728-733, Jun. 1996.

[9] P. S. Filipski, "Polyphase apparent power and power factor under distorted waveform conditions," IEEE Trans. Power Del., vol. 6, no. 3, pp. 1161-1165, Jul. 1991.

[10] A. E. Emanuel, "On the definition of power factor and apparent power in unbalanced polyphase circuits with sinusoidal voltage and currents," IEEE Trans. Power Del., vol. 8, no.3, pp. 841-852, Jul. 1993.

[11] P. S. Filipski, "Apparent power-A misleading quantity in the nonsinusoidal power theory:Are all nonsinusoidal power theories doomed to fail," Eur. Trans. Electr. Power Eng., vol. 3, no. 1, pp. 21-26, Jan./Feb. 1993.

[12] L. S. Czarnecki, "Comments on "Apparent and reactive powers in three phase systems: Insearch of a physical meaning and a better resolution'," Eur. Trans. Electr. Power Eng., vol.4, no. 5, pp. 421-426, 1994.

[13] G. T. Heydt, "Problematic power quality indices," in Proc. IEEE/PES Winter Meeting, Singapore, Jan. 2000, pp. 2838-2842. Panel Session - The need for future harmonic standards.

[14] E. J. Davis, A. E. Emanuel, and D. J. Pileggi, "Evaluation of singlepoint measurementsmethod for harmonic pollution cost allocation," IEEE Trans. Power Del., vol. 15, no. 1, pp.14-18, Jan. 2000.

[15] D.S. Dorr, "Point of utilization power quality study results," IEEE Transactions on Industry Applications, vol. 31, no. 4, pp. 658-666, 1995.

[16] IEC 61000-4-30, "Power Quality measurement standard".

[17] IEEE Recommended Practice for Monitoring Electric Power Quality.

[18] Daniel Sharon, Juan-Carlos Montaño, Antonio López, Antonio López, Dolores Borrás and Jaime Gutiérrez, Power Quality Factor for Networks Supplying Unbalanced Nonlinear Loads" IEEE Transactions on Instrumentation and Measurement, vol. 57, no. 6, june 2008 